\title{
Gastro-resistant Capsule, Soft Dosage Form
}

National Cancer Institute

\section{Source}

National Cancer Institute. Gastro-resistant Capsule, Soft Dosage Form. NCI Thesaurus.

Code C149532.

Solid single-dose, delayed-release preparation contained in a soft shell. The preparation is intended to resist the gastric fluid and to release the active substance(s) in the intestinal fluid. Soft gastro-resistant capsules are usually formed, filled and sealed in one operation. They may contain a liquid or semi-solid preparation in the gastro-resistant shell. Soft gastro-resistant capsules are intended for oral use. 